\title{
A case of mucinous borderline ovarian tumor with microinvasion that relapsed, with an adverse prognosis
}

\author{
Hiroaki Nagano $\cdot$ Mariko Fujibayashi · \\ Hideomi Sanai $\cdot$ Mitsue Muraoka $\cdot$ Koichiro Takagi
}

Received: 13 July 2011/Accepted: 13 October 2011/Published online: 17 December 2011

(C) The Japan Society of Clinical Oncology 2011

\begin{abstract}
The nomenclature and diagnostic criteria of mucinous borderline ovarian tumors (M-BOTs) are controversial. We report a case of M-BOT with microinvasion that relapsed, with an unfavorable outcome. A 40-year-old Japanese female underwent emergent laparotomy (left salpingo-oophorectomy) for a ruptured ovarian cystic tumor. The pathological diagnosis was M-BOT of the left ovary with microinvasion. She was followed up without additional therapy in accordance with her request, and 9 months after initial surgery the same tumor relapsed in the right ovary. She was surgically treated and followed up in the same manor as before. A second relapse occurred 25 months after the initial surgery; exploratory laparotomy revealed peritoneal dissemination. Biopsy confirmed diagnosis of mucinous adenocarcinoma. Although she received three biweekly cycles of a modified FOLFOX 6 regimen (oxaliplatin in combination with infusional 5-fluorouracil/ leucovorin) after surgery, she died of the disease 28 months after the initial surgery. Although the diagnostic criteria for M-BOT are debatable, cases of M-BOT with microinvasion may sometimes have adverse prognoses, as in this case.
\end{abstract}

Keywords Mucinous borderline ovarian tumor . Microinvasion · Intraepithelial carcinoma $\cdot$ Mucinous adenocarcinoma $\cdot$ FOLFOX

H. Nagano $(\varangle) \cdot$ H. Sanai · M. Muraoka $\cdot$ K. Takagi Department of Obstetrics and Gynecology, Tokyo Women's Medical University, Medical Center East, 2-1-10 Nishiogu, Arakawa-ku, Tokyo 116-8567, Japan

e-mail: naganoog@dnh.twmu.ac.jp

M. Fujibayashi

Department of Pathology, Tokyo Women's Medical University,

Medical Center East, 2-1-10 Nishiogu, Arakawa-ku,

Tokyo 116-8567, Japan

\section{Introduction}

Mucinous tumors account for $12-15 \%$ of all ovarian tumors. Mucinous borderline ovarian tumors (M-BOTs) and mucinous ovarian cancers (MOCs) account for $10-15 \%$ of all mucinous tumors [1]. In 2003, a workshop on borderline ovarian tumors was held in Bethesda, Maryland, USA, where the diagnostic criteria for defining various morphological groups within the M-BOT category, including borderline tumor with intraepithelial (noninvasive) carcinoma and borderline tumor with microinvasion, were discussed [2]. However, the nomenclature and diagnostic criteria of M-BOT remain controversial. We report the case of a 40-year-old Japanese woman with M-BOT accompanied by microinvasion, which recurred, with an adverse prognosis.

\section{Case report}

A 40-year-old para-2 Japanese woman with no relevant medical history was admitted to our medical center in October 2007 with a complaint of acute lower abdominal pain. On subsequent examination, a diagnosis of ruptured ovarian cystic tumor was made, and she underwent emergency laparotomy with left salpingo-oophorectomy. During the surgery, a frozen section was not available and the surgeons did not perform irrigation. The ruptured cystic tumor specimen of the left ovary measured approximately $8 \mathrm{~cm}$ in diameter, and was a single locule comprising a nest of smaller cysts surrounded by stroma (Fig. 1). Nine blocks from the specimen were microscopically examined. Papillary proliferation of the mucinous columnar epithelium of the intestinal type was observed in the cyst wall. There were two small foci of microinvasion, 1.5 and $1 \mathrm{~mm}$ in size. Adjacent to these foci, closely spaced cystic glands 


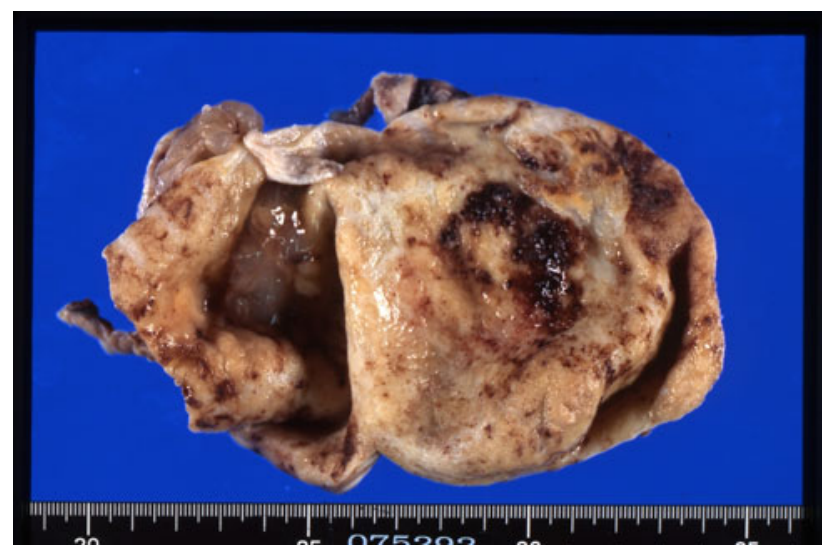

Fig. 1 Inner surface of the left (primary) ovarian tumor. A unilocular cyst bears numerous excrescences

lined by epithelial cells with marked nuclear atypia were observed (Fig. 2). The pathological diagnosis was M-BOT with microinvasion of the left ovary-The International Federation of Gynecology and Obstetrics (FIGO) stage Ic. The cytology of ascites was negative. The patient preferred a follow-up regimen with no further therapy, including staging laparotomy. We followed her up intensively with ultrasound after every 3 months.

The tumor recurred in the form of a multilocular cyst in the right ovary 9 months after the initial surgery. Secondary cytoreductive surgery was performed, and a multilocular mucinous cystic tumor of the right ovary was resected. Although there was no extra-ovarian lesion, hysterectomy and partial omentectomy were also performed for the purpose of staging. The peritoneal cavity was washed sufficiently with approximately $10,000 \mathrm{ml}$ pre-warmed saline. The resected tumor specimen was a multilocular cystic tumor comprising a large amount of mucinous fluid with a maximum diameter of $20 \mathrm{~cm}$. Twenty blocks from the tumor were microscopically examined. The cyst walls were lined with mucinous columnar epithelium of the intestinal type. Mild nuclear atypia and mitosis were observed in more than $10 \%$ of the specimens. The cell atypia was milder than that of the primary tumor (Fig. 3). The pathological diagnosis was M-BOT of the right ovary. There was no evidence of metastasis or involvement of the uterus, fallopian tube, or omentum. The post-surgical course was uneventful. The patient was followed up with no further treatment.

The second recurrence was observed 25 months after the initial surgery, when the patient presented with an elevated serum CA199 level of 724 U/l and massive ascites. Exploratory laparotomy revealed diffuse peritoneal disseminated lesions, each of which ranged in size from approximately $5-10 \mathrm{~mm}$, and approximately 2,400 ml mucinous ascites was evacuated. Several biopsy samples were taken from these lesions. Pathologically, the tumors had significant cell atypia, and destructive stromal infiltration by nests of malignant cells was observed (Fig. 4). Diagnosis of metastatic mucinous adenocarcinoma from the ovary was made. Having had informed consent, including the regimen not to be the standard therapy, she received chemotherapy with a modified FOLFOX 6 regimen: oxaliplatin $\left(85 \mathrm{mg} / \mathrm{m}^{2}\right)$ and leucovorin $\left(200 \mathrm{mg} / \mathrm{m}^{2}\right)$, both given as a 2 -h infusion, and 5-fluorouracil (5-FU: $400 \mathrm{mg} / \mathrm{m}^{2}$ ) given as an intravenous bolus on day 1 , followed by a $46-\mathrm{h}$ infusion of $5-\mathrm{FU}\left(2,400 \mathrm{mg} / \mathrm{m}^{2}\right)$, every 2 weeks from the tenth post-operative day. Although she received three cycles of this regimen, the disease could not be adequately controlled. She passed away on the 56th post-operative day, 28 months after the initial surgery.

\section{Discussion}

M-BOTs are of two distinct types: an intestinal type and a Mullerian (endocervical) type, which is relatively uncommon. Because our reported case presented with the intestinal type, the following discussion focuses on this type of M-BOT.

At least three difficulties are faced in respect of identifying the pathology of M-BOT of the intestinal type that may have affected the diagnosis and outcome of our case. First, primary ovarian mucinous tumors of the intestinal type may be extremely heterogeneous within an individual neoplasm. M-BOTs of the intestinal type often contain areas of cystadenoma and noninvasive carcinoma, and occult areas of invasive carcinoma may also coexist. Therefore, adequate sampling (a block of tissue for each $1-2 \mathrm{~cm}$ of the tumor's maximum dimension) of mucinous tumors is particularly important [3]. In our case, although an adequate number of samples was taken, there was a risk of missing a small focus of invasive malignancy.

The second difficulty lies in the pattern of microinvasion in M-BOTs. In up to $9 \%$ of M-BOT cases, one or more tiny foci of stromal invasion may be present $[4,5]$. The upper limit for the size of each individual focus varies from 1 to $5 \mathrm{~mm}$. A review of previous studies suggests 37 microinvasive tumors have been reported, and no recurrence or death due to the disease has been observed [2]. It has been suggested that microinvasion in M-BOTs should be classified into two categories: borderline tumor with microinvasion and borderline tumor with microinvasive carcinoma [4]. Foci of microinvasive carcinoma are of uncertain prognostic significance; when they are present, however, pathologists should search intensively for larger foci of invasive carcinoma. Our case was documented to have two small foci of microinvasion in the primary tumor, which may also be labeled microinvasive carcinoma according to this classification. 

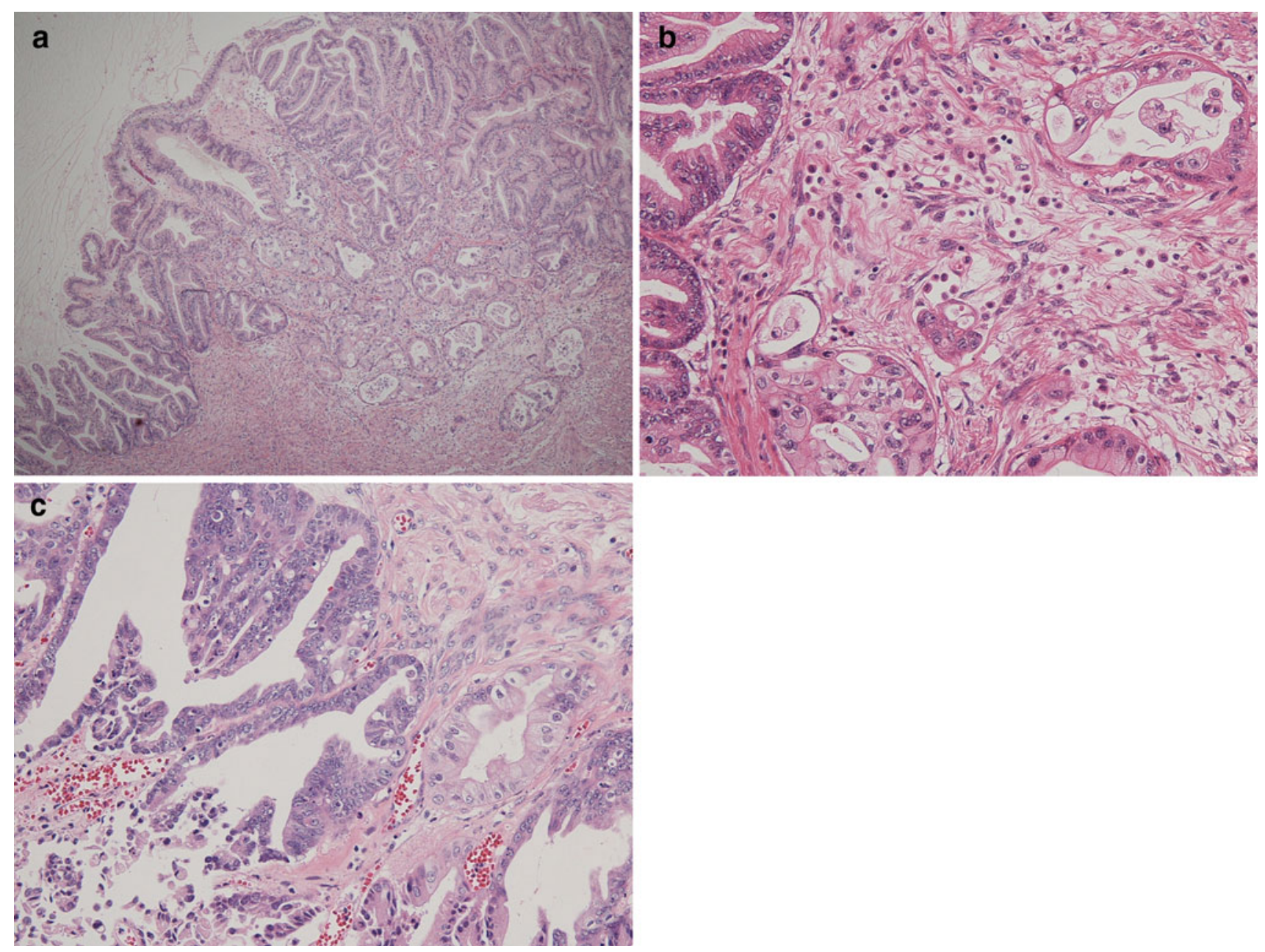

Fig. 2 Microscopic appearance of the left (primary) ovarian tumor. a The small foci of microinvasion can be seen. In the upper right area, closely spaced cystic glands lined by atypical epithelial cells can also be observed $(H \& E \times 40)$. b Higher power view of neoplastic

glands showing destructive stromal invasion $(H \& E \times 200)$. $\mathbf{c}$ The area adjacent to the field of stromal invasion. Neoplastic glands are lined by epithelial cells with considerable nuclear atypia $(\mathrm{H} \& \mathrm{E} \times 200)$
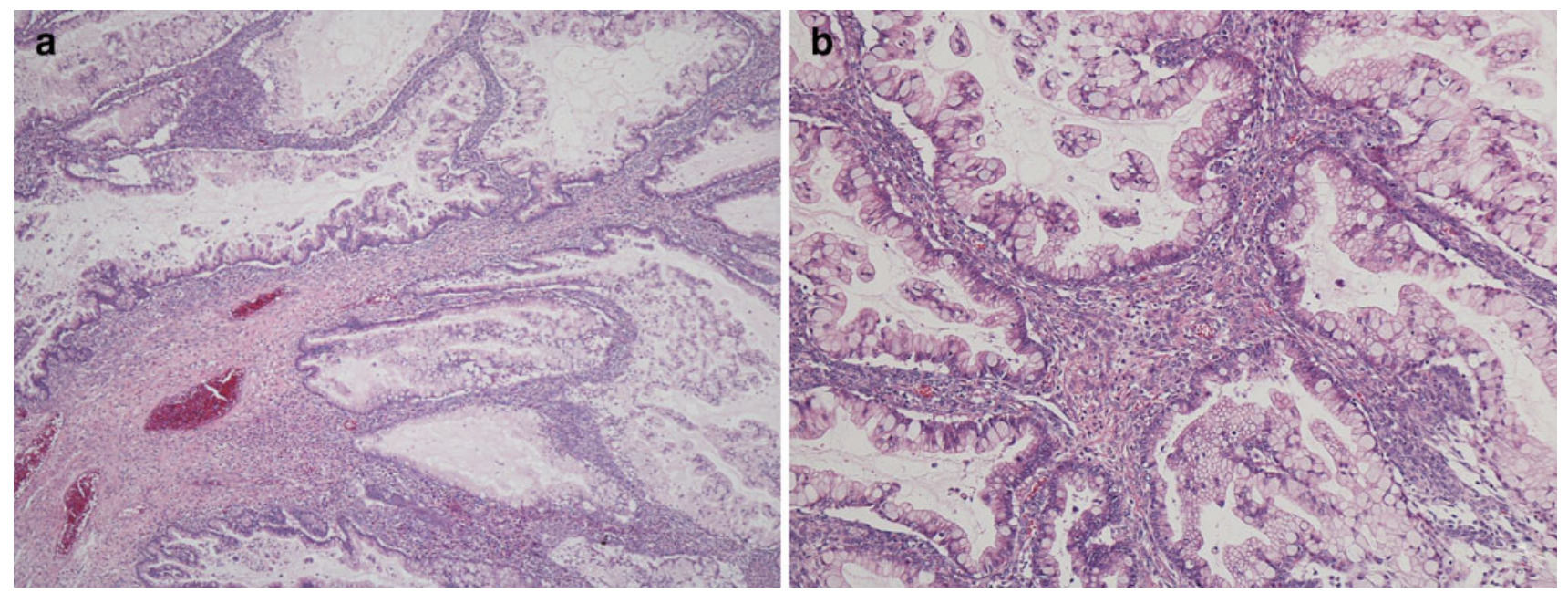

Fig. 3 Microscopic appearance of the recurrent tumor of the right ovary. a The cyst walls are lined with mucinous columnar epithelium of the intestinal type $(\mathrm{H} \& \mathrm{E} \times 40)$. b The cell atypia is milder than that of the primary tumor $(\mathrm{H} \& \mathrm{E} \times 100)$ 


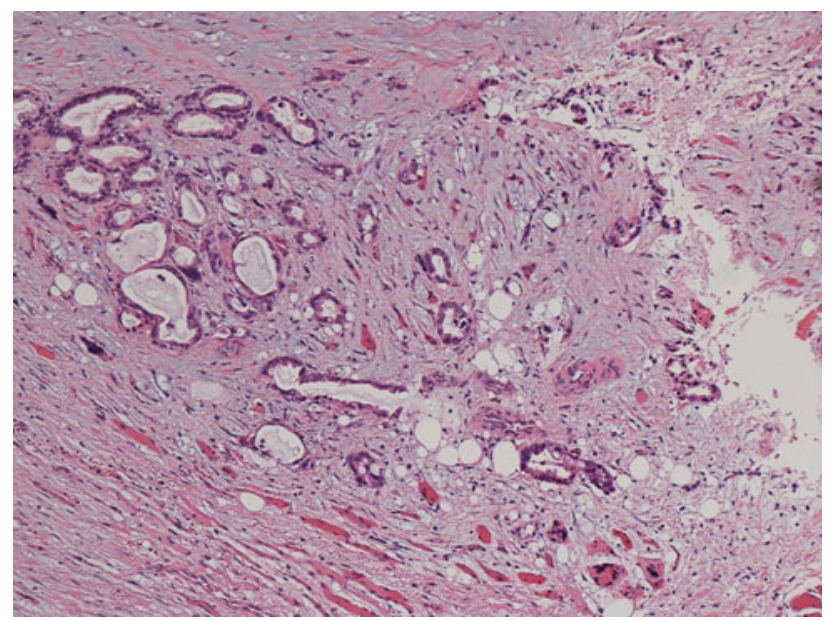

Fig. 4 Microscopic appearance of peritoneal dissemination at the second recurrence. The tumor is composed of neoplastic glands lying in a desmoplastic stroma $(\mathrm{H} \& \mathrm{E} \times 40)$

The third limitation is the difficulty in distinguishing between M-BOTs with intraepithelial carcinoma and invasive adenocarcinomas. Small or large areas of noninvasive mucinous carcinoma occur in approximately $15-55 \%$ of otherwise typical M-BOT cases [4, 5]. On the other hand, invasion in MOCs of the intestinal type can be either expansile (nondestructive) or infiltrative (destructive). It may be difficult to distinguish M-BOT with intraepithelial carcinoma from MOC with expansile invasion when, in the former, closely packed glands lined by histologically malignant epithelium are separated only by thin strands of stroma. There is significant interobserver variability among pathologists in this area [6]. The primary tumor in our case had the same microscopic features as those of M-BOT with intraepithelial carcinoma, including high-grade nuclear atypia (Fig. 2). On the basis of several studies identifying the type of invasion, it seems that adverse prognosis is mostly associated with the infiltrative rather than expansile type of invasion. Further studies of tumors with expansile invasion are warranted [2].

In late 2009, the Japan Society of Obstetrics and Gynecology and the Japanese Society of Pathology collaborated to publish a new version of the general rules for clinical and pathological management of ovarian tumors, mostly on the basis of the World Health Organization classification of 2003 [7, 8]. According to these rules, mucinous tumors with any stromal invasion are classified as carcinomas, because sufficient long-term observational data for mucinous tumors with microinvasion are not yet available. On the other hand, intraepithelial carcinoma still belongs to the borderline tumor category. Limitations of the pathological definition of M-BOT must still be addressed. Our case is apparently classified as MOC according to the new version of Japanese classification. As the phrase "M-BOT with microinvasion" could lead to confusion regarding its biological and prognostic significance, this new classification would be appropriate with regard to the adverse prognosis observed in this case. However, it is unclear as to which of the three problems mentioned above predominantly affected the prognosis in our case. Recently, Khunamornpong et al. [9] studied 171 cases of M-BOTs and reported 6 recurrent cases and 3 cases in which the patient died of the disease. They also pointed out the risk factors for recurrence of M-BOTs. All those factors were observed in our case: FIGO stage not less than Ic, microinvasion, age less than 45 years, and intraepithelial carcinoma.

MOC is known to be a chemo-resistant form of cancer. Taxane plus platinum, the standard regimen for common epithelial ovarian cancer, has only limited efficacy against this type of tumor [10]. Recently it has been noted that ovarian mucinous tumors have similar molecular biology to gastrointestinal malignancies [10, 11]. Several agents with chemotherapeutic efficacy against gastrointestinal malignancies have been tried, including oxaliplatin, capecitabine, and S-1. The FOLFOX regimen is currently one of the reference regimens for treatment of advanced colon/rectal cancer. Favorable results from several phase II studies involving the FOLFOX regimen for the treatment of recurrent ovarian cancer have been reported [12, 13]. For our case, we administered a modified FOLFOX 6 regimen, but could not improve the outcome for the patient. The Gynecologic Oncology Group (GOG) is planning to start a phase III clinical study of chemotherapy for MOC, in which an oxaliplatin plus capecitabine (XELOX) regimen and a paclitaxel plus carboplatin (TC) regimen will be compared (GOG 2041). Sato et al. [14] from Japan have confirmed the efficacy of a combination of oxaliplatin and 5-FU for MOCs in both in-vitro and invivo studies. They are starting a phase II study of the S-1 plus oxaliplatin (SOX) regimen for advanced or recurrent MOCs. Taxane plus platinum still remains the standard regimen for MOCs. We hope ongoing or further studies will identify the most promising treatments for these tumors.

Conflict of interest No author has any conflict of interest.

\section{References}

1. Scully RE, Young RH, Clement PB (1998) Tumors of ovary, maldeveloped gonads, fallopian tube and broad ligament. In: Atlas of tumor pathology, 3rd series, fascicle 23. Armed Forces Institute of Pathology, Washington

2. Ronnett BM, Kajdacsy-Balla A, Gilks CB et al (2004) Mucinous borderline ovarian tumors: points of general agreement and persistent controversies regarding nomenclature, diagnostic criteria, and behavior. Hum Pathol 35:949-960 
3. Hart WR, Norris HJ (1973) Borderline and malignant mucinous tumors of the ovary. Histologic criteria and clinical behavior. Cancer 31:1031-1045

4. Hart WR (2005) Borderline epithelial tumors of the ovary. Mod Pathol 18:33-50

5. Rodriguez IM, Prat J (2002) Mucinous tumors of the ovary: a clinicopathologic analysis of 75 borderline tumors (of intestinal type) and carcinomas. Am J Surg Pathol 26:139-152

6. McCluggage WG (2010) The pathology of and controversial aspects of ovarian borderline tumours. Curr Opin Oncol 22:462-472

7. Japan Society of Obstetrics and Gynecology, the Japanese Society of Pathology (ed) (2009) The general rules for clinical and pathological management of ovarian tumors, Part 1: histological classification and color atlas of ovarian tumors, 2nd edn. Kanehara-shuppan, Tokyo

8. Tavassoli FA, Devilee P (eds) (2003) World Health Organization Classification of Tumours: pathology and genetics of tumours of the breast and female genital organs. IARCP Press, Lyon

9. Khunamornpong S, Settakorn J, Sukpan K et al (2011) Mucinous tumor of low malignant potential ("borderline" or "atypical proliferative" tumor) of the ovary: a study of 171 cases with the assessment of intraepithelial carcinoma and microinvasion. Int $\mathbf{J}$ Gynecol Pathol 30:218-230

10. Hess V, 'Hern RA, Nasiri N et al (2004) Mucinous epithelial ovarian cancer: a separate entity requiring specific treatment. J Clin Oncol 22:1040-1044

11. Frumovitz M, Schmeler KM, Malpica A et al (2010) Unmasking the complexities of mucinous ovarian carcinoma. Gynecol Oncol 117:491-496

12. Pectasides D, Pectasides M, Farmakis D et al (2004) Oxaliplatin plus high-dose leucovorin and 5-fluorouracil (FOLFOX 4) in platinum-resistant and taxane-pretreated ovarian cancer: a phase II study. Gynecol Oncol 95:165-172

13. Rosa DD, Awada A, Mano MS et al (2008) Oxaliplatin/5-fluorouracil-based chemotherapy was active and well tolerated in heavily pretreated patients with ovarian carcinoma. Arch Gynecol Obstet 278:457-462

14. Sato S, Itamochi H, Kigawa J et al (2009) Combination chemotherapy of oxaliplatin and 5-fluorouracil may be an effective regimen for mucinous adenocarcinoma of the ovary: a potential treatment strategy. Cancer Sci 100:546-551 